\title{
Pterostilbene attenuates acute kidney injury in septic mice
}

\author{
YIZI XIA ${ }^{1}$, YING CHEN ${ }^{1}$, LUMING TANG $^{1}$, ZHENG WANG $^{1}$ and YU ZHENG ${ }^{2}$ \\ Departments of ${ }^{1}$ Emergency and ${ }^{2}$ Nephrology, The Second Affiliated Hospital of Wenzhou Medical University, \\ Wenzhou, Zhejiang 325027, P.R. China
}

Received February 27, 2017; Accepted October 27, 2017

DOI: $10.3892 / \mathrm{etm} .2018 .5808$

\begin{abstract}
Acute kidney injury (AKI) is a severe complication of sepsis with a high mortality and morbidity. Pterostilbene (Pte) has been suggested to confer anti-apoptotic and anti-inflammatory effects. In the current study, the effects of Pte on AKI were evaluated in septic mice. Cecal ligation and puncture surgery was performed to induce sepsis. The results suggested that Pte administration significantly decreased the levels of serum urea nitrogen and creatinine, and improved the survival rate of septic mice. Additionally, the renal injury induced by sepsis was attenuated by pterostilbene treatment. Notably, pterostilbene reduced $\mathrm{Bcl}-2$-associated $\mathrm{X}$ protein expression, and levels of interleukin- $1 \beta$ and tumor necrosis factor- $\alpha$, and upregulated B-cell lymphoma 2 expression. The results indicate that pterostilbene may serve as a potential therapeutic candidate for the treatment of AKI induced by sepsis.
\end{abstract}

\section{Introduction}

Sepsis is characterized by stimulation of a systemic inflammatory response related to an infection (1), usually complicated with multiple organ failure (MOF). Sepsis can lead to various complications. Acute kidney injury (AKI), as a common type of MOF, is a frequent and serious complication of sepsis in intensive care unit (ICU) patients (2), whose morbidity and mortality increase dramatically (3).

As sepsis-induced AKI is quite complicated, its pathophysiology has not been completely explicit and not fully understood. Emerging publications have shed light upon its underlying mechanisms, including the following aspects: i) renal macrocirculatory and microcirculatory disorder, ii) surge of inflammatory cytokines and oxidative stress, iii) coagulation cascade activation, and iv) bioenergetics adaptive response with controlled cell-cycle arrest aiming to prevent cell death (4). So it is critical to follow the pathogenesis of AKI in sepsis and then develop corresponding therapeutic

Correspondence to: Dr Yu Zheng, Department of Nephrology, The Second Affiliated Hospital of Wenzhou Medical University, 109 Xueyuan West Road, Wenzhou, Zhejiang 325027, P.R. China E-mail: zhengyu8359@sina.com

Key words: acute kidney injury, sepsis, pterostilbene, apoptosis approaches to treat sepsis-induced AKI, which can restore perfusion of the renal microcirculation, scavenge or suppress inflammatory markers and oxidative stress.

Pterostilbene (Pte), a natural analog of resveratrol, is a well-recognized antioxidant that primarily exists in blueberries, grapevines and heartwood of red sandalwood (5). And it has been suggested that the in vivo biological activity of equimolar doses of Pte may be greater than that of resveratrol (6), and the antioxidant activity of Pte are superior to resveratrol due to its higher lipophilicity, which increases its ability to be absorbed compared to resveratrol (6). Studies have demonstrated that resveratrol has salutary effect on sepsis-induced multiple organ dysfunction, such as myocardial depression (7), AKI (8-10). However, the effect of Pte on sepsis-induced AKI remains unclear.

Therefore, the aim of this study is to explore the potential protective role of Pte in the mouse model of sepsis-induced $\mathrm{AKI}$, and its potential underlying mechanisms.

\section{Materials and methods}

Reagents. Pte was purchased from Sigma-Aldrich (St. Louis, MO, USA). Mouse serum creatinine (Scr) assay kit was purchased from Crystal Chem Inc. (Downers Grove, IL, USA). Mouse blood urea nitrogen (BUN) detection kit was purchased from Bio-Medical Assay Co., Ltd (Beijing, China). Mouse tumor necrosis factor-alpha (TNF- $\alpha$ ) and Interleukin-1 beta (IL-1 $\beta$ ) ELISA kits were purchased from Thermo Fisher Scientific Inc. (Waltham, MA, USA). The rabbit polyclonal antibody against B-cell lymphoma 2 (Bcl-2) and Bcl-2associated protein $\mathrm{x}$ (Bax) was purchased from Santa Cruz Biotechnology (Santa Cruz, CA, USA). Ultrapure RNA kit was obtained from Takara Biotechnology Co., Ltd (Dalian, China).

Animals. Male C57BL/6J mice aged 6-8 weeks were purchased from the Laboratory Animal Center of Wenzhou Medical University. The mice were kept at $25^{\circ} \mathrm{C}$ (humidity $50 \%$ ) and $12 \mathrm{~h}$ light/ $12 \mathrm{~h}$ dark cyclical alternates. This study was performed according to the Guide for the Care and Use of Laboratory Animals, which is published by the US National Institutes of Health (National Institutes of Health Publication no. 85-23, revised 1996) and was approved by the Ethics Committee of Wenzhou Military Medical University.

Animal model of cecal ligation and puncture (CLP). Sepsis was induced by CLP as previously reported (11). Briefly, mice 
were anesthetized by $50 \mathrm{mg} / \mathrm{kg}$ pentobarbital sodium (i.p.) and a midline incision $(1-2 \mathrm{~cm})$ was made to expose the internal organs. The cecum was ligated and punctured with a 20-gauge needle in two places, and a small amount of cecal contents was expressed through the puncture wound to induce sepsis. The bowel was then sent back to the abdomen, and the incision was sutured with a sterile $6-0$ silk. The mice in the sham-operated group underwent the same operation without CLP.

Experimental groups and drug treatment. Mice were randomly divided into the following three groups: The Sham group, CLP + vehicle group, CLP + Pte $15 \mathrm{mg} / \mathrm{kg}$ group. Pte was first dissolved in DMSO and then diluted in normal saline and then Pte was administered intraperitoneally twice $(0.5$ and $4 \mathrm{~h}$ after CLP induction). The CLP + vehicle group was administered the same volume of vehicle. $24 \mathrm{~h}$ after CLP induction, all the animals were euthanized and peripheral blood and kidney tissues were collected for further experiments.

Evaluation of survival rate. The 7-day survival rate was evaluated after CLP induction. After the survival study, animals were euthanized upon exhibition of certain symptoms/features i.e., discomfort/pain.

Histopathological examination. Kidney samples were collected $24 \mathrm{~h}$ after CLP induction and fixed in 10\% formalin solution. Then the samples were embedded in paraffin and sectioned into $5-\mu \mathrm{m}$-thick sections. Sections were stained with hematoxylin and eosin (H\&E) and assessed under light microscopy at magnification, $\mathrm{x} 200$. Sections were examined to evaluate the degree of pathological changes based on glomerular epithelial hyperplasia, tubular dilatation and protein cast formation, and inflammatory cell infiltrations. These were scored from 0 to 3 (normal to severe, total score was 0 to 9). The total score for each sample was evaluated.

Assessment of renal function. Renal functions were evaluated by detecting BUN and Scr levels with commercially available kits according to the manufactures' instructions.

Detection of inflammatory cytokines. Kidney tissues from different groups were homogenized with PBS on ice, and then centrifuged at $4^{\circ} \mathrm{C}$ for $40 \mathrm{~min}$ at $12,000 \mathrm{rpm}$. Subsequently, the supernatants were collected to perform ELISA. The levels of inflammatory cytokines, including TNF- $\alpha$ and IL- $1 \beta$ in kidney tissue were detected by commercially available ELISA kits according to the manufacturer's recommendation.

$R T$ - $q P C R$. Total RNA was extracted using Ultrapure RNA kit according to the manufacturer's instructions. Total RNA $(400 \mathrm{ng})$ was then reverse transcribed. Amplification conditions were $95^{\circ} \mathrm{C}(10 \mathrm{~min})$ followed by 50 cycles of $95^{\circ} \mathrm{C}(30 \mathrm{sec})$ and $60^{\circ} \mathrm{C}(20 \mathrm{sec})$. Primers were as follows: Bax forward, 5'-CAGGATGCGTCCACCAAGAA-3' and reverse, 5'-AGT AGAAGAGGGCAACCACG-3'; Bcl-2 forward, 5'-GAGTAC CTGAACCGGCATCT-3' and reverse, 5'-GGTATGCAC CCAGAGTGATG-3'; and GAPDH forward, 5'-AAGAGG GATGCTGCCCTTAC-3' and reverse 5'-TACGGCCAAATC CGTTCACA-3'. The amount of mRNA for each gene was

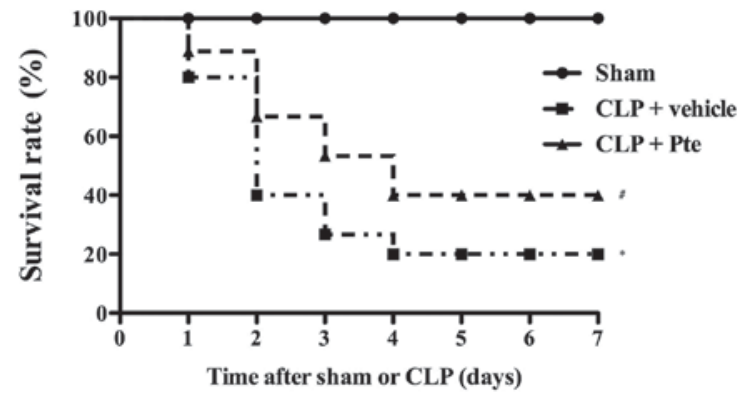

Figure 1. Effect of Pte treatment on the survival rate of mice after CLP. Mice were monitored daily for 7 days. Values are expressed as survival percentage, $\mathrm{n}=20$ in each group. ${ }^{*} \mathrm{P}<0.05$ vs. Sham group, ${ }^{\#} \mathrm{P}<0.05$ vs. CLP + vehicle group. CLP, cecal ligation and puncture; Pte, pterostilbene; Pte, pterostilbene.

normalized by GAPDH, and the relative expression levels were calculated using the $2^{-\Delta \Delta \mathrm{Ct}}$ method (12). Relative mRNA levels of each gene in the Sham group samples were adjusted to 1 . The mRNA levels in the experimental groups were expressed as fold-changes as compared with that of the Sham samples.

Western blot analysis. The kidney samples were collected and protein extractions were obtained. Total proteins from renal lysates $(50 \mu \mathrm{g})$ were separated by $10 \%$ sodium dodecyl sulfate-polyacrylamide gels electrophoresis (SDS-PAGE), and transferred to polyvinylidene fluoride (PVDF) membranes. The membranes were then blocked with $5 \%$ non-fat milk for $2 \mathrm{~h}$ at room temperature, followed by incubating with primary antibodies against $\mathrm{Bcl}-2$ and $\mathrm{Bax}$ at $4^{\circ} \mathrm{C}$ overnight. $\beta$-actin was used as the loading control. Membranes were developed with ECL western blotting substrate (GE Healthcare, Buckinghamshire, UK) and western blots were visualized and analyzed on the Bio-Rad Image System (Bio-Rad, Berkeley, CA, USA).

Statistical Analysis. All analyses were performed using GraphPad Prism 6 (GraphPad Software, La Jolla, CA). Data are expressed as mean \pm standard error of the mean (SEM) and compared via one-way analysis of variance (ANOVA) followed by Bonferroni multiple comparisons test. Survival rate was determined by the Kaplan-Meier estimator and compared by a log-rank test. $\mathrm{P}<0.05$ was considered to indicate statistically significant difference.

\section{Results}

Pte treatment improves survival rate after CLP induction. To investigate the protective effect of Pte against lethality induced by CLP induction, we first evaluated the 7-day survival rate in the three groups. As shown in Fig. 1, the 7-day survival rate in sham group was almost $100 \%$. However, 7 days after CLP surgery, the survival rate decreased dramatically (vs. the sham group, $\mathrm{P}<0.05)$. Pte administration the significantly increased survival rate in the CLP + Pte group (vs. the CLP group, $\mathrm{P}<0.05)$.

Pte attenuates the histopathological injury of kidney in septic mice. Histopathological evaluation was performed using $\mathrm{H} \& \mathrm{E}$ 
A

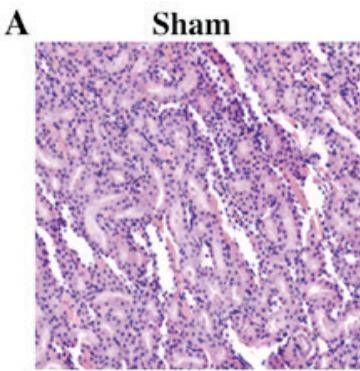

B
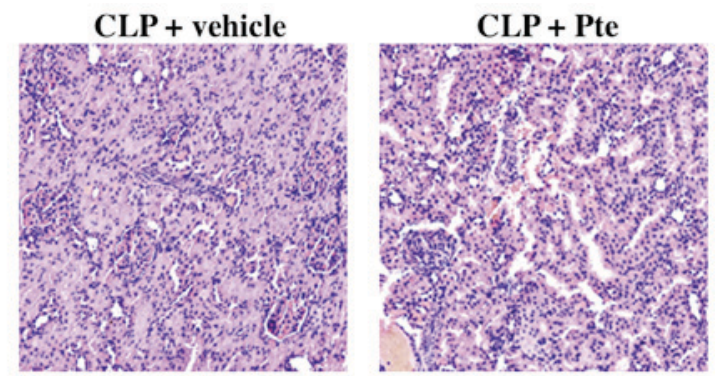

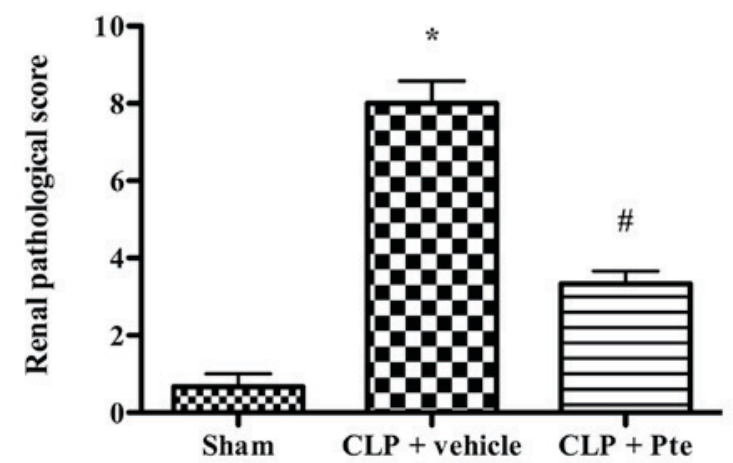

Figure 2. The effect of Pte on renal pathology in septic mice. (A) Representative images of renal histopathologic changes (magnification, x200). (B) Pathological scores of renal tissues of each group. Data are expressed as mean $\pm \mathrm{SEM}, \mathrm{n}=6$ in each group. ${ }^{*} \mathrm{P}<0.05$ vs. Sham group, ${ }^{\#} \mathrm{P}<0.05$ vs. CLP + vehicle group. CLP, cecal ligation and puncture; Pte, pterostilbene; SEM, mean \pm standard error of the mean.
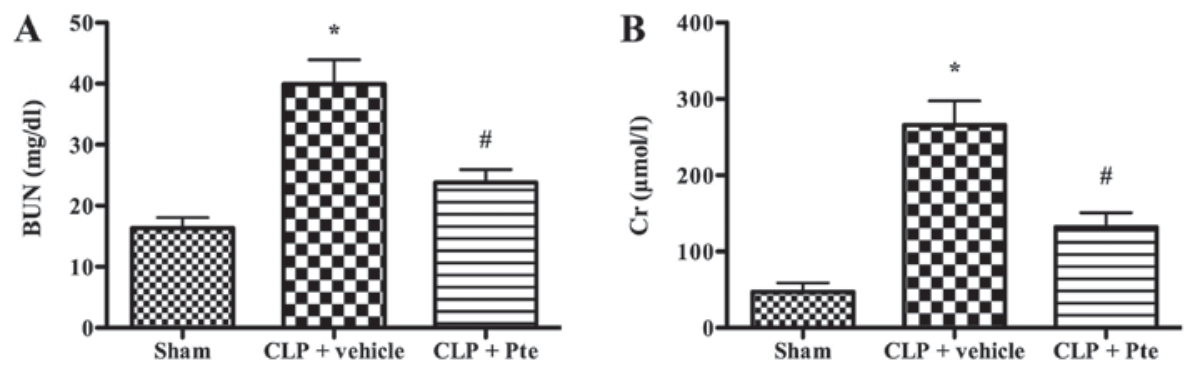

Figure 3. Effect of Pte treatment on the levels of BUN and Scr in mice after CLP. (A) BUN level. (B) Scr level. Data are expressed as mean \pm SEM, $n=6$ in each group. ${ }^{*} \mathrm{P}<0.05$ vs. Sham group, ${ }^{\#} \mathrm{P}<0.05$ vs. CLP + vehicle group. Scr, serum creatinine; CLP, cecal ligation and puncture; Pte, pterostilbene; SEM, mean \pm standard error of the mean.

staining (Fig. 2). In the Sham group, the renal tissue was intact. Histologic evaluation of the kidney in the CLP + vehicle group indicated severe pathological changes, including glomerular epithelial hyperplasia, tubular dilatation, abundant protein exudation, and numerous inflammatory cell infiltrations in the renal interstitium. Pte treatment significantly attenuated the severity of pathological changes as compared with the CLP + vehicle group.

Pte reduces Scr and BUN levels after CLP. Scr and BUN levels are regarded as critical indicators of renal function. The levels of BUN and Scr were dramatically elevated after CLP (Fig. 3). In Pte-treated mice, the levels of BUN and Scr reduced dramatically compared to the CLP + vehicle group $(\mathrm{P}<0.05)$.

Pte suppresses inflammatory response in vivo. To investigate the effect of Pte on inflammatory response in vivo, representative inflammatory cytokines were determined by ELISA. As shown in Fig. 4, the levels of TNF- $\alpha$ and IL-1 $\beta$ were higher in sepsis group $(\mathrm{P}<0.05)$. Pte treatment significantly decreased the levels of TNF- $\alpha$ and IL-1 $\beta(\mathrm{P}<0.05)$.

Effect of Pte on apoptosis-related proteins expression after CLP. To further investigate the effects of Pte on apoptosis-related proteins expression in the renal tissue of septic mice, the expression of $\mathrm{Bax}$ and $\mathrm{Bcl}-2$ in was analyzed using qRT-PCR and western blot (Fig. 5). Our data indicated that the expression of Bax mRNA and protein markedly decreased in the Pte-treated group $(\mathrm{P}<0.05)$ compared with the CLP + vehicle group. The expression of Bcl-2 mRNA and protein significantly increased in the Pte-treated group $(\mathrm{P}<0.05)$. This result suggested that Pte confers anti-apoptotic effect against AKI induced by CLP.

\section{Discussion}

In the current study, we established a CLP-induced sepsis model to evaluate the effects of Pte on sepsis-induced AKI. CLP is the most widely used animal model of sepsis and it was 

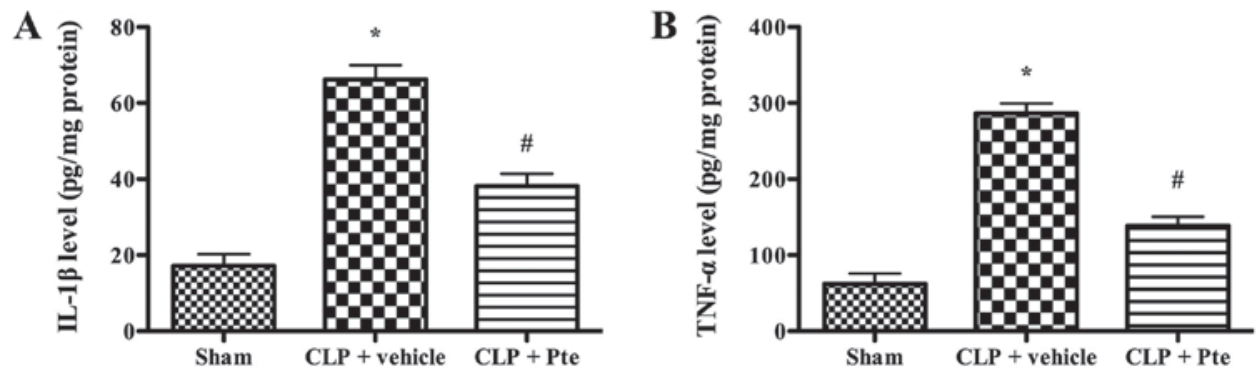

Figure 4. Effect of Pte treatment on the levels of IL- $1 \beta$ and TNF- $\alpha$ in mice after CLP. (A) IL-1 $\beta$ level. (B) TNF- $\alpha$ level. Data are expressed as mean \pm SEM, $n=6$ in each group. ${ }^{*} \mathrm{P}<0.05$ vs. Sham group, ${ }^{~} \mathrm{P}<0.05$ vs. $\mathrm{CLP}+$ vehicle group. CLP, cecal ligation and puncture; Pte, pterostilbene; SEM, mean \pm standard error of the mean. TNF- $\alpha$, tumor necrosis factor-alpha; IL-1 $\beta$, Interleukin-1 beta.
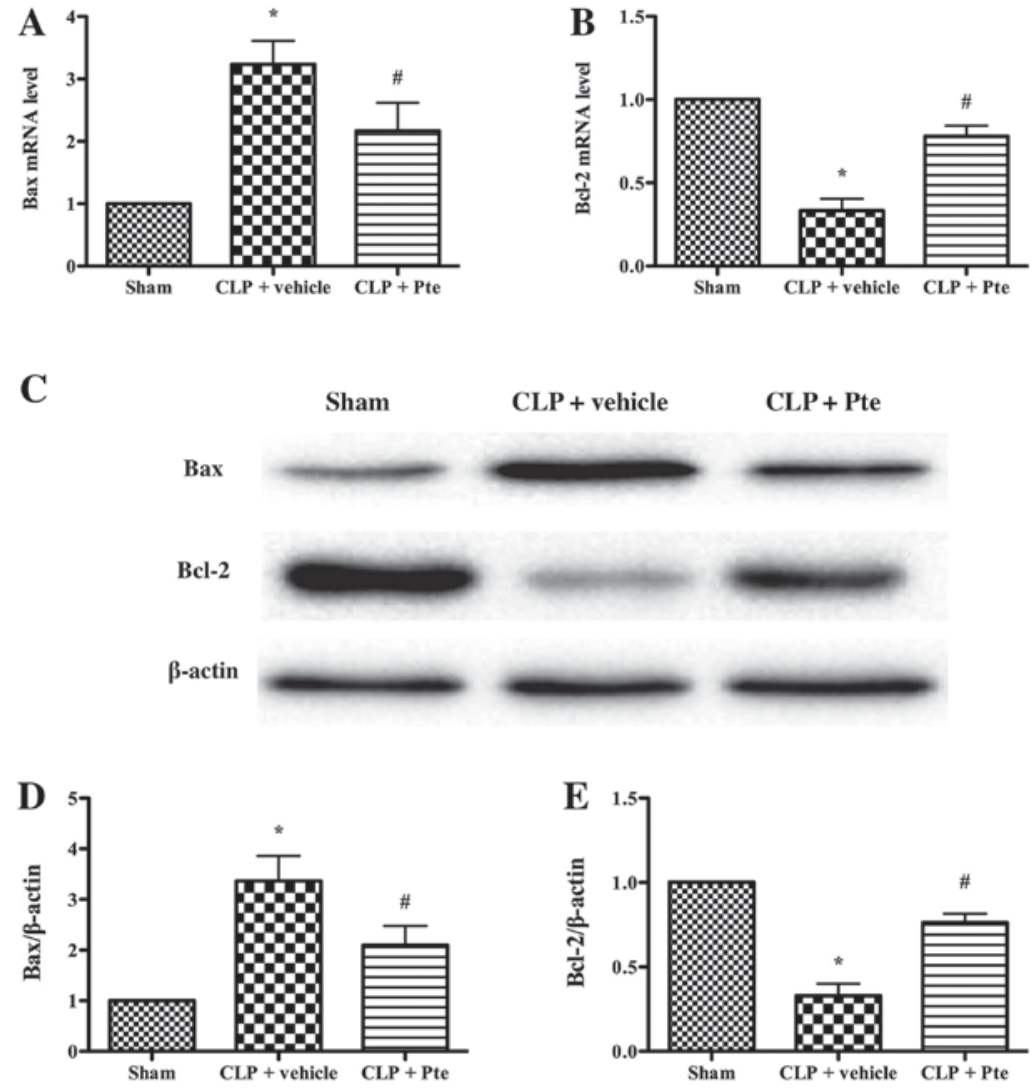

Figure 5. Effect of Pte treatment on the expression of Bax and Bcl-2. (A) Bax mRNA level. (B) Bcl-2 mRNA level. (C) Representative images of western blot

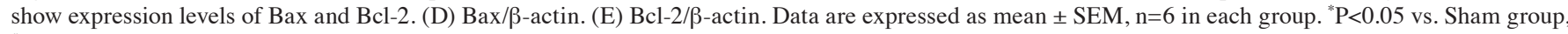
${ }^{\#} \mathrm{P}<0.05$ vs. CLP + vehicle group. CLP, cecal ligation and puncture; Pte, pterostilbene; SEM, mean \pm standard error of the mean. Bcl-2, B-cell lymphoma 2; Bax, Bcl-2-associated protein $\mathrm{x}$.

considered as a reliable and clinically relevant animal model of the human sepsis (13). The major findings in the present study are: (1) Pte treatment improves the survival rate in mice subjected to CLP surgery. (2) Pte treatment attenuates kidney injury in septic mice, as evidenced by the lowered pathological changes and decreased BUN and Scr levels. (3) Pte treatment suppresses inflammatory response and apoptosis in septic mice.

We first evaluate whether Pte treatment can improve the survival rate in septic mice. Our results suggest that Pte treatment significantly improves 7-day survival rate after CLP induction, indicating the protective effects of Pte against sepsis. Then, we evaluate the effect of Pte on the pathological changes of kidney. Sepsis results in obvious inflammatory cells infiltration in renal interstitium, glomeruli and nephric tubules. This injury can be attenuated dramatically by Pte treatment. The BUN and Scr levels increase significantly in mice subjected to CLP, indicating the severe injury of the kidney. Pte treatment alleviates kidney injury as evidenced by the decreased levels of BUN and Scr levels.

Sepsis-induced AKI accounts for nearly 50\% of cases of AKI in the ICU (14). Moreover, AKI is the leading cause of death in the ICU (15). Although previous studies have suggested that hypotension, renal vasoconstriction and ischemia-reperfusion injury as the primary pathophysiological mechanisms involved in sepsis-induced AKI, recent studies have focused on other 
pathophysiological mechanisms, including microcirculatory flow abnormalities, inflammation, cell bioenergetic adaptive responses to injury, microparticles, which are involved in septic AKI (16). Inflammation triggered by sepsis leads to the activation of leukocytes and release of pro-inflammatory cytokines. Pro-inflammatory cytokines, especially IL-1 $\beta$ and TNF- $\alpha$, damage the main function of vascular endothelial cells, which plays a critical role in maintaining the fluidity of blood. In addition, IL-1 $\beta$ and TNF- $\alpha$ enhance clot formation by impairing the anticoagulant pathway via the inhibition of the production of activated protein C (17). In the current study, our results suggest that Pte treatment dramatically decrease the levels of IL- $1 \beta$ and TNF- $\alpha$, indicating the anti-infalmmatory effect of Pte in AKI induced by sepsis. It has been reported that Pte exerts protection against sepsis-induced liver injury (18). Liu et al (18) suggested that Pte decrease the infiltration of neutrophil into the liver. So, neutrophil may be, at least, one of the target cells of Pte.

Finally, we evaluate the effect of Pte on renal apotosis induced by sepsis. Apoptosis is a programmed cell death, and it is involved in sepsis induced kidney injury (19). Apoptosis is regulated by the anti-apoptotic family and the pro-apoptotic family. Bcl-2, known as a key protein in the anti-apoptotic family, is localized in the outer membrane of mitochondria, and it plays an important role in promoting cellular survival and inhibiting the actions of pro-apoptotic proteins (20). It has been suggested that $\mathrm{Bcl}-2$ can inhibit the release of cytochrome c, which can trigger apoptosis, from the mitochondria (21). On the contrary, Bax is a key protein in the pro-apoptotic family. Bax can promote the permeabilization and release of cytochrome $\mathrm{c}$ and ROS, resulting the activation of the apoptosis cascade (22). In the current study, our data indicates that CLP induces a dramatic increase in Bax expression and a decrease in $\mathrm{Bcl}-2$ expression in the kidney, which were alleviated by Pte treatment.

In summary, Pte exerts protective effects via suppressing inflammation and apoptosis in sepsis-induced AKI in mice. The current study suggests that administration of Pte may be a potential management for the treatment of sepsis. However, the precise mechanisms still need to be further elucidated before its clinical application.

\section{References}

1. van der Poll T and Opal SM: Host-pathogen interactions in sepsis. Lancet Infect Dis 8: 32-43, 2008.

2. Bagshaw SM, George C and Bellomo R; ANZICS Database Management Committee: Early acute kidney injury and sepsis: A multicentre evaluation. Crit Care 12: R47, 2008.

3. Kissoon N, Daniels R, van der Poll T, Finfer S and Reinhart K: Sepsis-the final common pathway to death from multiple organ failure in infection. Crit Care Med 44: e446, 2016.
4. Shum HP, Yan WW and Chan TM: Recent knowledge on the pathophysiology of septic acute kidney injury: A narrative review. J Crit Care 31: 82-89, 2016.

5. McCormack D and McFadden D: A review of pterostilbene antioxidant activity and disease modification. Oxid Med Cell Longev 2013: 575482, 2013.

6. Kapetanovic IM, Muzzio M, Huang Z, Thompson TN and McCormick DL: Pharmacokinetics, oral bioavailability, and metabolic profile of resveratrol and its dimethylether analog, pterostilbene, in rats. Cancer Chemother Pharmacol 68: 593-601, 2011.

7. Smeding L, Leong-Poi H, Hu P, Shan Y, Haitsma JJ, Horvath E, Furmli S, Masoom H, Kuiper JW, Slutsky AS, et al: Salutary effect of resveratrol on sepsis-induced myocardial depression. Crit Care Med 40: 1896-1907, 2012.

8. Holthoff JH, Wang Z, Seely KA, Gokden N and Mayeux PR: Resveratrol improves renal microcirculation, protects the tubular epithelium, and prolongs survival in a mouse model of sepsis-induced acute kidney injury. Kidney Int 81: 370-378, 2012.

9. Sebai H, Ben-Attia M, Sani M, Aouani E and Ghanem-Boughanmi N: Protective effect of resveratrol in endotoxemia-induced acute phase response in rats. Arch Toxicol 83: 335-340, 2009.

10. Chen L, Yang S, Zumbrun EE, Guan H, Nagarkatti PS and Nagarkatti M: Resveratrol attenuates lipopolysaccharide-induced acute kidney injury by suppressing inflammation driven by macrophages. Mol Nutr Food Res 59: 853-864, 2015.

11. Chen LW, Chen W, Hu ZQ, Bian JL, Ying L, Hong GL, Qiu QM, Zhao GJ and Lu ZQ: Protective effects of growth arrest-specific protein 6 (Gas6) on sepsis-induced acute kidney injury. Inflammation 39: 575-582, 2016.

12. Livak KJ and Schmittgen TD: Analysis of relative gene expression data using real-time quantitative PCR and the 2(-Delta Delta C(T)) method. Methods 25: 402-408, 2001.

13. Doi K, Leelahavanichkul A, Yuen PS and Star RA: Animal models of sepsis and sepsis-induced kidney injury. J Clin Invest 119: 2868-2878, 2009.

14. Umbro I, Gentile G, Tinti F, Muiesan P and Mitterhofer AP: Recent advances in pathophysiology and biomarkers of sepsis-induced acute kidney injury. J Infect 72: 131-142, 2016.

15. Hutchins NA, Unsinger J, Hotchkiss RS and Ayala A: The new normal: Immunomodulatory agents against sepsis immune suppression. Trends Mol Med 20: 224-233, 2014.

16. Pettilä V and Bellomo R: Understanding acute kidney injury in sepsis. Intensive Care Med 40: 1018-1020, 2014.

17. Weiler $\mathrm{H}$ and Ruf W: Activated protein $\mathrm{C}$ in sepsis: The promise of nonanticoagulant activated protein C. Curr Opin Hematol 15: 487-493, 2008

18. Liu X, Yang X, Han L, Ye F, Liu M, Fan W, Zhang K, Kong Y, Zhang J, Shi L, et al: Pterostilbene alleviates polymicrobial sepsis-induced liver injury: Possible role of SIRT1 signaling. Int Immunopharmacol 49: 50-59, 2017.

19. Gómez H and Kellum JA: Sepsis-induced acute kidney injury. Curr Opin Crit Care 22: 546-553, 2016.

20. Liu H, Zhao L, Yue L, Wang B, Li X, Guo H, Ma Y, Yao C, Gao L, Deng J, et al: Pterostilbene attenuates early brain injury following subarachnoid hemorrhage via inhibition of the NLRP3 inflammasome and Nox2-related oxidative stress. Mol Neurobiol: Sep 24, 2016 (Epub ahead of print).

21. Jemmerson R, Dubinsky JM and Brustovetsky N: Cytochrome C release from CNS mitochondria and potential for clinical intervention in apoptosis-mediated CNS diseases. Antioxid Redox Signal 7: 1158-1172, 2005.

22. Luna-Vargas MP and Chipuk JE: Physiological and pharmacological control of BAK, BAX, and beyond. Trends Cell Biol 26: 906-917, 2016. 